\title{
Reflection plate evaluation for the invisible area measurements using infrared thermography
}

\author{
Sang Chae Kim¹, Hyun Chul Jung², Man Yong $\mathrm{Choi}^{3}$, Kyung Suk Kim \\ ${ }^{1}$ Department of Mechanical System Engineering, Chosun University, Gwangju, Korea , \\ bluerune@nate.com \\ ${ }^{2}$ Department of Mechanical System Engineering, Chosun University, Gwangju,Korea , \\ hyunchul.jung@chosun.ac.kr \\ ${ }^{3}$ Korea Research Institute of Standards and Science (KRISS), Daejeon,Korea, \\ mychoi@kriss.re.kr \\ ${ }^{4}$ Department of Mechanical System Engineering, Chosun University, Gwangju,Korea , \\ gsckim@chosun.ac.kr
}

\section{Introduction}

Defect inspection system for industrial applications takes the important portion, and non-contact inspection method has been significantly improved. Infrared thermograph, as one of method for non-contact inspection, can provide relatively precise data and quick inspection time. In addition, temperature distribution and thermal variation can be acquired. Therefore, it is widely applied to detect the defect out of temperature change.

Infrared thermography presents real-time images of temperature variation by detecting radiation energy from object surface. It has been used for thermal characteristic evaluation, thermal imaging on power system, defect inspection for composite material, evaluation for building insulation, medical diagnosis using body temperature, etc. [1]

In addition, needs for defect detection method have been increased to prevent industrial and economic loss including injury due to material and equipment damage in power plant or chemical industries. [2, 3]

Infrared thermography utilizes heat source from object, and it has the benefits of non-hazardous, quickness, multiple streams, broad scanning, etc. [4]

Purpose of this study is to compensate the issues at defect measurement on non-accessible area of rear section of pipe or where camera cannot be accessible by selecting proper reflector using temperature change analysis.

\section{Background of infrared thermography}

All objects, which are above absolute temperature, emit IR radiation energy. IR thermography is used to detect and measure thermal energy in IR spectrum from object. These IR energies are originated from vibration and rotation of molecule. As temperature of object is high, more movement is followed, and higher amount of infrared energy is radiated. This energy is detected by infrared thermography camera. IR thermography camera utilizes device to detect IR spectrum from object, and converts the amount of IR to temperature. It can be displayed as IR or thermal image. Therefore, IR thermography can show temperature distribution on the surface of object, and it can give temperature distribution at each point.

IR detector is different by spectrum used, and it is classified as near, mid, and far IR.

\section{Experimental method}


Because temperature can vary according to IR thermography cameras, halogen lamps, and reflector materials, influence of reflector can be determined using the system listed in Fig. 1. As shown in Fig. 2, aluminum, brass, glass, copper, carbon steel, pure iron, and wafer in $100 \mathrm{~mm} \times 100 \mathrm{~mm} \times 10 \mathrm{~T}$ are selected as candidates. In addition, SC5500 from FLIR is used to measure mid IR wavelength.

Temperature of reflector after heat absorption is measured with IR thermography camera by heating with halogen lamp. Distance between IR thermography camera, halogen lamp, and reflector is $1 \mathrm{~m}$ each, and reflector is heated up to 5 minutes with halogen lamp. After that, temperature is measured for 3 times per each material.

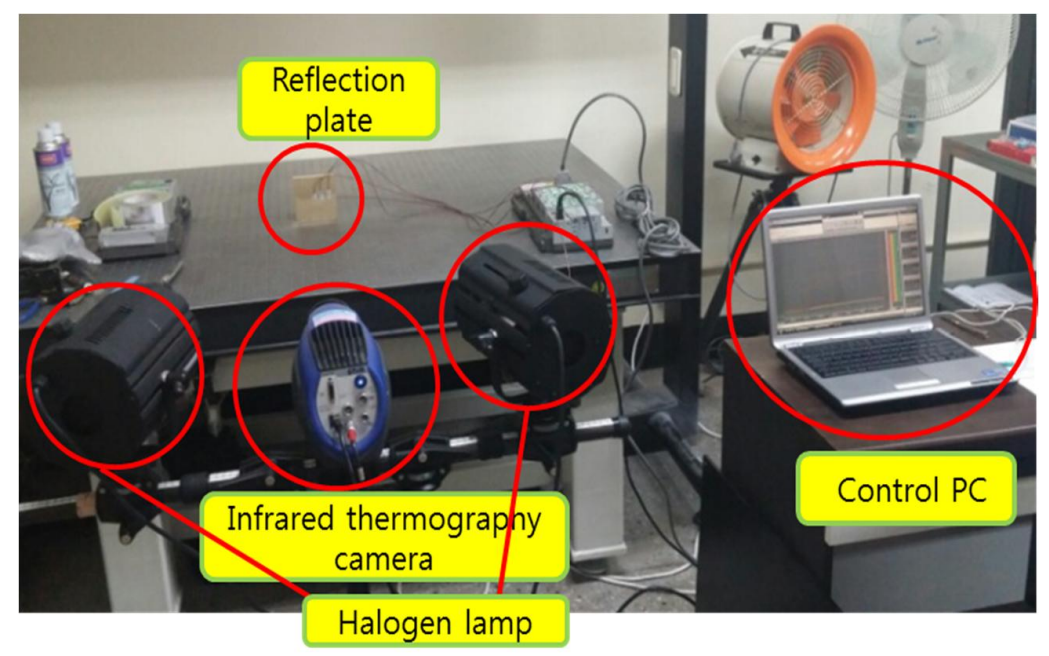

Fig 1. Infrared Thermography system

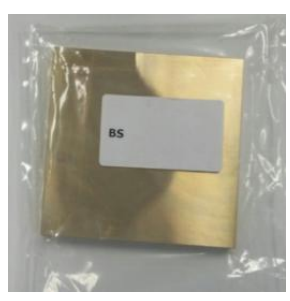

(a) Brass

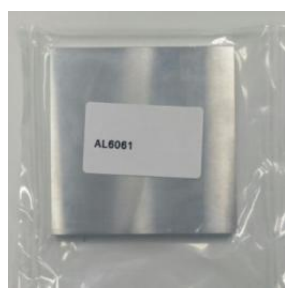

(e) Aluminum

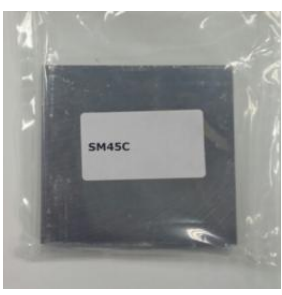

(b) Carbon steel

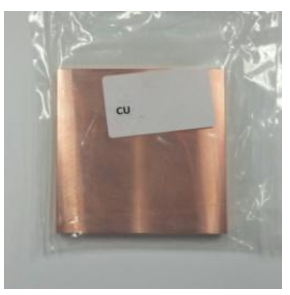

(c) Copper

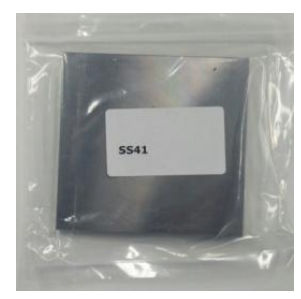

(d) Pure iron

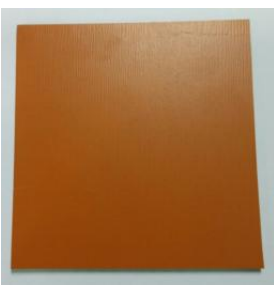

(f) Glass

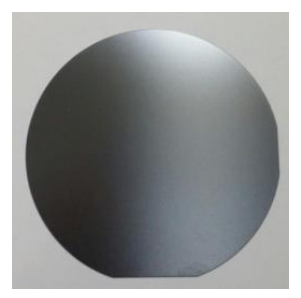

(g) Wafer

Fig 2. Reflector 


\section{Experimental results}

Using IR thermography camera, temperature change of reflector is confirmed as Table 1, energy variation and heat transfer rate from reflector, which absorbs thermal energy from halogen lamp, is shown in Table 2.

As shown in Fig. 3, pure iron showed the largest temperature change, and higher content of iron also indicates bigger variation of energy. In addition, glass shows higher response to temperature, but energy absorption is low from defect measurement. Therefore, it is not suitable for reflector.

Higher energy variation can ensure higher absorption of thermal energy, and it will increase the chance to detect defect in target.

Table 1. The slope of the temperature change per unit time of the reflector ( $d T / d t)$

\begin{tabular}{|c|c|c|c|c|}
\hline & 1 time & 2 time & 3 time & Average \\
\hline \hline Aluminum & 0.013462 & 0.011795 & 0.010427 & 0.011894 \\
\hline Brass & 0.010101 & 0.010526 & 0.008939 & 0.009855 \\
\hline Glass & 0.085465 & 0.081675 & 0.074757 & 0.080633 \\
\hline Copper & 0.008173 & 0.006796 & 0.005769 & 0.006913 \\
\hline Carbon Steel & 0.023645 & 0.024658 & 0.018182 & 0.022162 \\
\hline Pure iron & 0.035751 & 0.023585 & 0.019807 & 0.026381 \\
\hline Wafer & 0.027632 & 0.035366 & 0.040476 & 0.034491 \\
\hline
\end{tabular}

Table 2. Thermal conductivity and Energy change of the reflector

\begin{tabular}{|c|c|c|}
\hline & Thermal conductivity & Energy change \\
\hline \hline Aluminum & 0.237 & 2.886465 \\
\hline Brass & 0.1 & 3.201688 \\
\hline Glass & 0.00057 & 2.539927 \\
\hline Copper & 0.4 & 2.403127 \\
\hline Carbon Steel & 0.043 & 8.088525 \\
\hline Pure iron & 0.045 & 9.247381 \\
\hline Wafer & 0.149 & 0.218303 \\
\hline
\end{tabular}




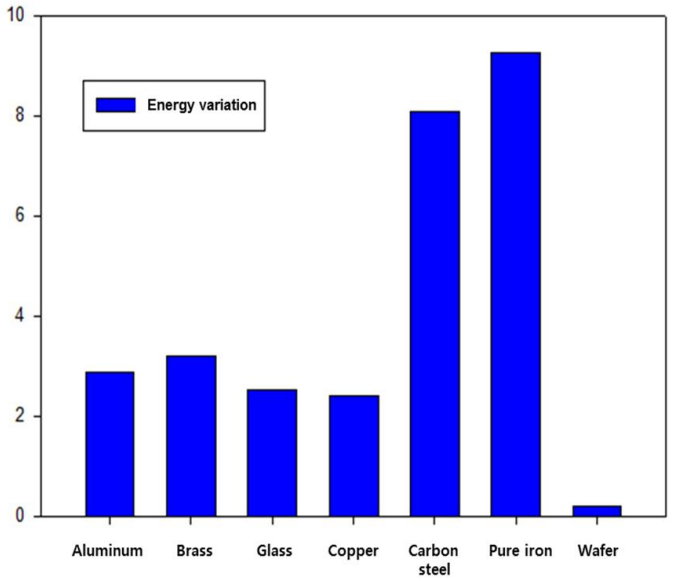

(a) Energy variation Graph

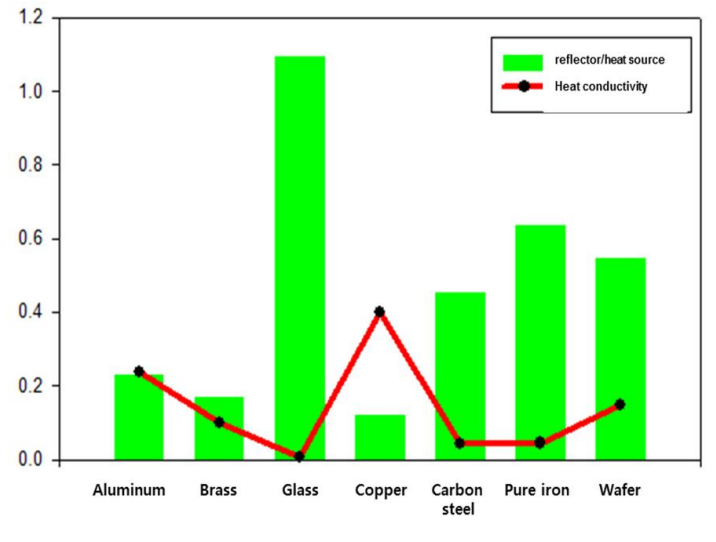

(b) Heat conductivity and ratio of reflector/heat source

Fig 3. Result Graph

\section{Conclusions}

In this study, IR thermography system and reflector is used to detect defect at the non-accessible location, and the appropriate reflector material is evaluated. Based on temperature change and energy variances for each reflector, metallic materials, such as pure iron, carbon steel, aluminum, and brass, are considered to be better. Proper material can be selected based on space limitation and thermal characteristics of target material, detection rate of defect can be improved.

For further study, detection of defect in non-accessible area using the candidate materials for reflector will be conducted.

\section{Acknowledgement}

This work was supported by the National Research Foundation of Korea(NRF) grant funded by the Korea government(MSIP) (No. 2012M2A2A9035705)

This work was supported by the National Research Foundation of Korea(NRF) grant funded by the Korea government(MSIP) (No. 2012-0668)

\section{REFERENCES}

[1] G. Gaussorgurs, "Infrared thermography" Translated by S. Chomet, Champmam \& Hall.London, pp.415-452(1994)

[2] M. Y. Choi, " The utilization of nondestructive testing and defects diagnosis using infrared thermography ," Journal of the Korean Society for Nondestructive Testing, Vol. 24, No.5, pp.525531 (2004)

[3] Y. M. Jung, "Experimental evidence and analysis of a mode conversion of guided wave using magnetostrictive strip transducer," Journal of the Korean Society for Nondestructive Testing, Vol. 29, No. 2, pp. 93-97 (2009) 
http://dx.doi.org/10.21611/qirt.2015.0084

[4] N. K. D. Grande and P. F. Durbin, "Using emissivity-corrected thermal maps to locate deep structural in concrete bridge decks", SPIE Symposium Report, pp. 124-137 V. M. Malhotra and N. J. Carino, "Non- destructive Testing of Concrete, "2nd ed., CRC press, pp. 15-1-15-14,(2004) 